\title{
Signaling Changes in Reinforcer Ratios Facilitates Adaptive Forgetting in Pigeons
}

\author{
Daniel Bell-Garrison
}

Follow this and additional works at: https://researchrepository.wvu.edu/etd

\section{Recommended Citation}

Bell-Garrison, Daniel, "Signaling Changes in Reinforcer Ratios Facilitates Adaptive Forgetting in Pigeons" (2016). Graduate Theses, Dissertations, and Problem Reports. 5180.

https://researchrepository.wvu.edu/etd/5180

This Thesis is protected by copyright and/or related rights. It has been brought to you by the The Research Repository @ WVU with permission from the rights-holder(s). You are free to use this Thesis in any way that is permitted by the copyright and related rights legislation that applies to your use. For other uses you must obtain permission from the rights-holder(s) directly, unless additional rights are indicated by a Creative Commons license in the record and/ or on the work itself. This Thesis has been accepted for inclusion in WVU Graduate Theses, Dissertations, and Problem Reports collection by an authorized administrator of The Research Repository @ WVU. For more information, please contact researchrepository@mail.wvu.edu. 
Signaling Changes in Reinforcer Ratios Facilitates Adaptive Forgetting in Pigeons

\section{Daniel Bell-Garrison}

Thesis submitted to the Eberly College of Arts and Sciences at West Virginia University in partial fulfillment of the requirements for the degree of

Master of Science

in

Psychology

Elizabeth G. E. Kyonka, Ph.D., Chair

Michael Perone, Ph.D.

Natalie Shook, Ph.D.

Department of Psychology

Morgantown, West Virginia

2016

Keywords: choice; pigeons; rapid acquisition; adaptive forgetting

Copyright 2016 Daniel Bell-Garrison 


\section{Abstract \\ Signaling Changes in Reinforcer Ratios Facilitates Adaptive Forgetting in Pigeons \\ Daniel Bell-Garrison}

Forgetting is often characterized as maladaptive, but if a cue no longer signals the consequences of a response, then forgetting the previously learned stimulus-response discrimination is adaptive. Pigeons pecked for food in concurrent schedules of reinforcement. The relative frequency of food delivery on each key changed pseudorandomly across sessions with an overnight break in the middle of each session. New sessions began immediately after the last food delivery in the previous session. When the change from one session to the next was not signaled, responses remained under the control of the previous session's ratio of reinforcement. When the session change was signaled by a change in the color of the keylights, control by the ratio from the previous session was diminished. Without interference from past ratios, sensitivity to the ratio of reinforcement was greater in the signaled than the unsignaled condition. This decrease in sensitivity to past ratios marks an example of adaptive forgetting. 
Table of Contents

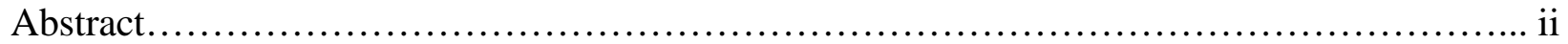

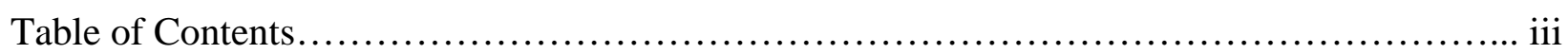

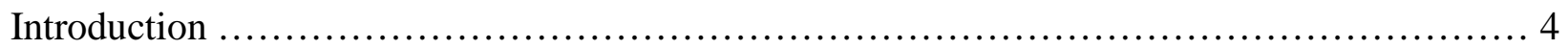

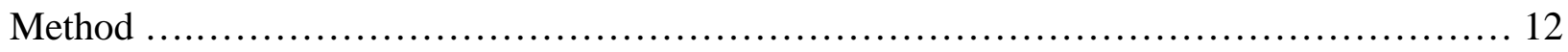

Results ....................................................................... 17

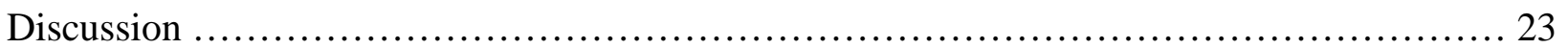

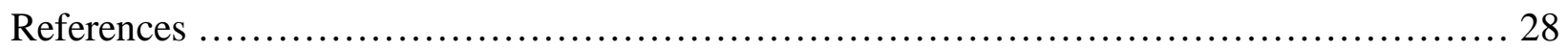

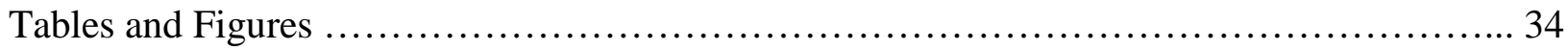


Signaling Changes in Reinforcer Ratios Facilitates Adaptive Forgetting in Pigeons

Animals of all kinds, humans and non-humans alike, continually choose between multiple alternatives when seeking reinforcement, whether that be food, water, social contact, or finishing a homework assignment. Most natural environments that animals face are dynamic; the value of an alternative can change relative to the value of other available alternatives. For example, driving on 705 is normally the fastest way to get to University Town Center from Suncrest Town Centre. However, during a home football game it would be faster to take a route that avoids the dense traffic. The choices that individuals make change in accordance with changes in the environment. Cues that signal those changes, such as a calendar of home football games, may help choice adapt to changing contingencies. The present study was conducted to evaluate how choice adapts to signaled and unsignaled changes in contingencies of reinforcement.

Researchers using what are called “rapid acquisition” operant-conditioning procedures have found that choice can adapt to dynamic changes in contingencies of reinforcement (Grace \& Nevin, 1999, Grace, Bragason, \& McLean, 2003, Kyonka \& Grace 2007, 2008, 2010). These experimenters have used procedures wherein the contingencies of reinforcement change unpredictably across sessions. With enough training under these conditions, responses are initially allocated evenly between the two alternatives. This shows that pigeons forget past contingencies. After some experience with the contingency on each alternative, more responses are allocated to the alternative that produces the food faster. In other words, once pigeons have experienced several reinforced trials from each alternative, they peck the key that produces the shorter time to food. 
While this line of research has shown that choice can adapt to unpredictable changes across sessions when those sessions also occur across calendar days, to date no studies have investigated the extent to which the circadian structure of the session controls changes in choice. Control by contingencies does not carry over across sessions, and there are at least two possible explanations for this result. One is that the overnight break is too long a retention interval for pigeons to recall prior contingencies from the previous day. An alternative possibility is that the break is a cue for the pigeons to forget the past ratios. If the overnight break is a cue, then it stands to reason that other types of cues may be able to facilitate adaptive forgetting. This study was conducted to answer three research questions related to adaptive forgetting. 1) How does response allocation on a concurrent schedule adapt when sessions are run back-to-back with no break between them? 2) To what extent do contingencies retain control over choice following an overnight break in the middle of the session? 3) How does cueing the change from one session to the next impact the way in which choice adapts?

\section{Adaptive Forgetting}

Although forgetting is often considered to be maladaptive, Kraemer \& Golding (1997) postulated that many instances of forgetting are beneficial because it enables animals to respond to the present environment more appropriately. Kraemer and Golding defined adaptive forgetting as "forgetting in the absence of a failed process" (p. 480). For instance, when someone gets a new cell phone number it is beneficial to forget the old cell phone number. When a cue no longer signals the consequences of a response, continuing to respond to the previous contingency does not maximize value. Forgetting the previously learned discrimination could be more adaptive.

Researchers have observed adaptive forgetting in a variety of animals and in various environments. Animals that live in dynamic environments, where the location of foraging areas 
or predators changes frequently, tend to stop responding to relevant cues more quickly than animals in less dynamic environments. Epp (2013) found that wild salamanders had less intense predator avoidance responses compared to lab-reared salamanders. The salamanders that had experienced wild environments did not respond to cues signaling potential predators, and this could be adaptive because the wild salamanders may have learned to balance predator avoidance with foraging or other survival behavior. Tadpoles similarly forget, or fail to show avoidant behavior toward, cues for low-risk predators more quickly than high-risk predators (Ferrari \& Chivers, 2013). Further, variables such as growth rate have been found to interact with adaptive forgetting. Brown et al. (2011) found that rainbow trout with higher growth rates did not retain recognition of (i.e., they forgot) predator cues faster than trout with lower growth rates. When cues change frequently it can be adaptive to forget those cues quickly, and research shows that evolutionarily successful animals do just that.

The way in which sessions are arranged in operant-conditioning research may be relevant to the study of adaptive forgetting. A session is a period of time in which behavior is measured and antecedents or consequences for the behavior are controlled (Johnston \& Pennypacker, 2009). In some areas of behavior analysis, particularly in applied research, many sessions may be run in a row followed by breaks of variable durations. By contrast, in non-human laboratory experiments, sessions typically occur once per day at about the same time every day. The circadian structure of an experiment may control responding to some degree. When testing sessions are conducted daily at the same time every day for the duration of the experiment, they are separated by a long overnight break. That break could serve as a cue for adaptive forgetting. The primary objective of the present experiment was to determine whether the circadian 
structure of an experiment was a necessary or sufficient cue for choice to adapt to a dynamic environment.

\section{Measuring choice}

Choice is defined as the allocation of time or behavior to one alternative among two or more available options (Baum \& Rachlin, 1969). Concurrent schedules, as opposed to trialbased procedures, are often used in experimental analyses of choice because they permit continuous measurement of response allocation. A concurrent schedule involves establishing two simultaneously available alternatives in which responding occasionally produces a reinforcer. One variant of this procedure is a concurrent variable interval (VI) VI schedule (Herrnstein, 1961). On a VI schedule, a reinforcer is available after an unpredictable interval of time. Once the interval has elapsed, one response is required for the reinforcer to be collected. In a common version of a concurrent VI VI schedule used with pigeons called an interdependent concurrent schedule (Stubbs \& Pliskoff, 1969), two illuminated keys are available for the pigeon to peck. However, at any one time, only one of the keys is active in that an interval is timing down or response-contingent reinforcement is available. As the interval times down for the left key, for example, no timer is arranged for the right key. Once the interval has elapsed and a response occurs, the reinforcer is delivered. A computer program then randomly selects the next active key. Arranging the VI schedules in this way ensures that the obtained rates of reinforcement on each key are very similar to the programmed rates. Additionally, measures of response allocation are relatively unconstrained by this procedure because it does not interfere with the opportunity to respond on either key.

In an interdependent concurrent VI VI schedule, choice is the ratio of responses allocated to the left and right keys. Nearly 50 years of research has found that the relation between the 
allocation of responses and the reinforcement available from two alternatives is well-described by a power function (Baum, 1974, 1979; Staddon, 1968, see Grace \& Hucks, 2013 for a recent review). This function has been observed in many experimental procedures involving a wide variety of experimental conditions (Wearden \& Burgess, 1982, Davison \& McCarthy, 1988). Experimentation and naturalistic observation across many species have established this relation as a reliable function (see Poling, Edwards, Weeden, \& Foster, 2011 for a recent review).

The power function described above is known as the generalized matching law. The relative allocation of responses between two alternatives will match the relative rate of reinforcement available upon those alternatives (Baum, 1974, 1979). The generalized matching law can be written as

$$
\log \frac{B_{L}}{B_{R}}=a \log \frac{R_{L}}{R_{R}}+\log b
$$

where $B_{L}$ is response rate on the left key, $B_{R}$ is response rate on the right key, and $R_{L}$ and $R_{R}$ are the rates of reinforcement on the left and right keys, respectively. For ease of interpretation, the generalized matching law is typically log-transformed because when a power function is logtransformed, the transformed relation is a linear function. The parameter $a$ represents sensitivity and describes the extent to which the ratio of reinforcement is controlling behavior. Higher sensitivity values show higher control by the contingencies. The parameter $b$ represents bias and accounts for what Baum (1974) considered to be unknown experimental error that results in responses being allocated to an alternative for reasons other than rate of reinforcement. Sensitivity and bias are derived from response allocation directly. Sensitivity is the slope of the linear model. If response allocation did not vary systematically with the rate of reinforcement, the slope of the model would be close to zero. If relatively more responses are allocated to the left or right key when the rate of reinforcement favors the respective alternative, then the slope of 
the model will be greater than zero. The intercept of the line is the logarithm of the bias term in Equation 1. If relatively more responses are allocated to the left or right key regardless of the ratio of reinforcement, responding is biased toward one of the alternatives. Since bias and sensitivity are independent of each other, sensitivity can be compared across subjects even when there is considerable intersubject variability in estimates of bias.

\section{Choice in transition}

Behavior analytic researchers rely on steady-state procedures, wherein behavior is maintained on some contingency of reinforcement for a lengthy period of time, because this method allows researchers to clearly observe effects of their independent variable upon their dependent variables. While it is critically important to evaluate behavior in steady-state experiments, transitions between steady-states occur both in the lab and in natural environments. Although dynamic environments may not easily lend themselves to steady-state procedures, it is still important to observe stable and replicable transitions in order to elucidate the functional relations between environmental variables and transitioning behavior (Sidman, 1960). One way to examine stable responses to dynamic environments is to change the contingencies of reinforcement in an unpredictable way at frequent, regular intervals. This approach is called “rapid acquisition” (Grace, Bragason, \& McLean, 2003) because the contingencies of reinforcement rapidly acquire control over behavior every day.

Schofield and Davison (1997) manipulated schedules of reinforcement daily to examine how response allocation adapts to a dynamic environment. In their experiment, pigeons keypecked on a concurrent VI VI schedule until 40 reinforcers were delivered in each daily session. The arranged reinforcer ratios for the schedule were changed pseudorandomly every session. The rate of reinforcement ratio was 1:2, 1:4 or 1:8, depending on the condition. From session to 
session, the key associated with the shorter VI schedule changed unpredictably. For example, a concurrent VI 37.4-s VI 150-s schedule (1:4 reinforcer ratio) was arranged during one session, but a concurrent VI 150-s VI 37.4-s (4:1 ratio) was in place during the next session.

Theoretically, when reinforcer ratios change frequently, pigeons may remember past contingencies such that control over response allocation by those past contingencies may carry over from one session to successive sessions. Schofield and Davison (1997) used an extended version of the generalized matching law to include reinforcer ratios from previous sessions in order to account for current-session behavior when ratios change frequently:

$$
\log \frac{B_{0 L}}{B_{0 R}}=a_{0} \log \frac{R_{0 L}}{R_{0 R}}+a_{1} \log \frac{R_{1 L}}{R_{1 R}}+\ldots+a_{9} \log \frac{R_{9 L}}{R_{9 R}}+\log b
$$

The response ratio in a given session is determined by the reinforcer ratio from the current and prior sessions. The variables are the same as in Equation 1 except that the subscripts represent the session from which the ratio was in effect, with 0 for the current session and $1 \ldots 9$ for previous sessions. This equation can be extended to include as many sessions as desired. Among researchers who use the method of rapid acquisition, Equation 2 is referred to as a lag analysis. The term 'lag' refers to the session from which reinforcer ratios are selected relative to the session in which responding occurred. Sensitivity to ratios that are lagged 0 sessions $\left(a_{0}\right)$ is referred to as Lag 0 sensitivity. Sensitivity to ratios lagged by 1 or more sessions (e.g., $a_{1}$ ) are referred to according to the appropriate lag (e.g., Lag 1 sensitivity).

After pigeons in Schofield and Davison’s (1997) experiment had a month of training with contingencies that changed every day, response allocation was sensitive to the contingencies of reinforcement by the end of every session. Lag 0 sensitivity values were between .70 and .81 . These values were comparable to previous concurrent VI VI research, where the contingencies 
remained the same across sessions (Myers \& Myers, 1977, Baum, 1979). Estimates of sensitivity to reinforcer ratios from previous sessions $\left(a_{1}-a_{9}\right)$ were close to zero. Responding repeatedly came under the control of each session's reinforcer ratios within a session, and reinforcer ratios from previous sessions had little effect on current session response allocation. In other words, pigeons adaptively forgot past ratios of reinforcement because control over response allocation did not carry over from one session to successive sessions.

A similar procedure that has been used to study response allocation is a concurrent chain schedule. In one variation of the concurrent chain schedule used to study choice between delayed rewards, the subject first responds during a choice phase that resembles a concurrent VI VI schedule; this is called the initial link. A response that satisfies the schedule on one of the initial link alternatives produces access to one of two mutually-exclusive outcome schedules that end in reinforcement (the terminal links). The initial links are often VI schedules of equal values (e.g. VI 10-s VI 10-s) and the terminal links are fixed interval (FI) schedules of differing lengths (e.g. FI 10-s and FI 20-s). On a FI schedule, the first response after a fixed amount of time will produce a reinforcer. For example, the initial links may both be VI 10-s schedules. A response that satisfies the schedule on the left key may lead to a FI 10-s schedule, but the right key may lead to a FI 20-s schedule. Since $10 \mathrm{~s}$ is more immediate than $20 \mathrm{~s}$, the matching law would predict that more responses would be allocated to the left initial link key because it is associated with more immediate access to food.

Grace, Bragason, and McLean (2003) found that when the terminal link delays in a concurrent chain were changed unpredictably across sessions, response allocation during the initial links became sensitive to the relative immediacy of food in the terminal links. Grace et al. arranged a concurrent chain schedule in which both of the initial links were always VI 10-s 
schedules, but the relative duration of the terminal links changed. The left terminal link was always a FI 8-s schedule, whereas the right terminal link alternated pseudo-randomly across sessions between a FI 16-s and a FI 4-s schedule. Grace et al. used a lag analysis (Equation 2) to find that choice for the shorter terminal link was rapidly acquired within a single session. Lag 0 sensitivity gradually increased over the course of a session, whereas sensitivity to ratios lagged greater than 0 was near zero for the entirety of the sessions. Control by relative terminal link immediacy was acquired by the end of a session and immediacy ratios from past sessions were forgotten (i.e., response allocation was not sensitive to past ratios).

This finding, that the current session's terminal link immediacy ratios control response allocation during the initial link, is reliable across many variations of the concurrent chain procedure (Grace \& McLean, 2006, Kyonka \& Grace, 2007, 2009, 2010). In each of these studies, choice was controlled by terminal link FI schedules from the current session, whereas contingencies from previous sessions had very little control over responding.

In the present experiment, I used a concurrent VI VI schedule to investigate the role of signaling in choice adaptation. Rates of food delivery from the two alternatives changed every session. To determine how response allocation adapts when sessions are run back-to-back with no break between them, I examined sensitivity (see Equation 1) of response allocation to the relative rate of food delivery throughout the session. To quantify the extent that reinforcement ratios retained control over choice following an overnight break in the middle of the session, I compared response ratios immediately before and after the overnight break. Finally, to determine how cueing the change from one session to the next impacted the way in which choice adapts, I analyzed changes in Lag 0 and Lag 1 sensitivity (see Equation 2) when a signal accompanied or did not accompany the change in sessions. 


\section{Method}

\section{Subjects}

Four White Carneau pigeons (Columba livia) were maintained at 85\% ad libitum weight $\pm 15 \mathrm{~g}$ through appropriate daily feedings after they were returned to their home cages. Pigeons were housed individually in cages with a 12-hr:12-hr light:dark cycle with free access to water. All pigeons had between 235 and 304 sessions of experience with minimal-variation rapidacquisition concurrent chains (see Kyonka \& Grace, 2007) in previous experiments (Subramaniam \& Kyonka, 2016).

\section{Apparatus}

Four operant-conditioning chambers (25.5 cm deep x 32 cm wide x 33.5 cm high) enclosed in sound-attenuating boxes containing ventilation fans that provided air circulation and white noise were used. Each chamber contained three keys located $24 \mathrm{~cm}$ above the floor arranged in a row $6 \mathrm{~cm}$ apart; the side keys were $2.5 \mathrm{~cm}$ from the walls of the chamber. A force of approximately $0.15 \mathrm{~N}$ was necessary to operate each key. A houselight, located on the back wall at the top of the chamber, provided general illumination at all times, except during the delivery of food, during all phases of the procedure. Food hoppers, each with a $5.5 \mathrm{~cm} \mathrm{x} 6 \mathrm{~cm}$ aperture centered $5.5 \mathrm{~cm}$ above the floor when activated, were filled with Nutriblend pigeon

pellets. The hopper was illuminated during the delivery of food. To ensure that pigeons had access to at least $10 \mathrm{~g}$ of food per session, each food delivery consisted of three hopper presentations of $2.5 \mathrm{~s}$ each, with $.5 \mathrm{~s}$ in between presentations. A computer and MED-PC® interface located in an adjacent room controlled the sessions.

\section{Procedure}


Because all pigeons had previous experience with rapid-acquisition concurrent chains, no pre-training was deemed necessary before the start of the current experiment. In each session, the left and right keys were illuminated and an interdependent concurrent VI 20-s VI 20-s schedule (Stubbs \& Pliskoff, 1969) was arranged upon the side keys. The rate of food delivery for each key was randomly selected at the start of each session. The side keys were illuminated white for the entire session in the unsignaled condition. Key color alternated between green and red across sessions in the signaled condition. These conditions will be described in more detail below. Figure 1 shows a procedural diagram for the unsignaled condition; the signaled condition was the same except for the color of the keys.

\section{Interdependent VI schedule}

A list of 12 intervals, each selected without replacement from an exponentially distributed (Fleshler \& Hoffman, 1962) list of intervals with a mean of 20 s, was used to determine when food would be made available. After each food delivery, the computer selected an interval from the list and selected the left or right key to be the active key. After the selected interval had elapsed, the first peck to the selected side key produced food (provided a 1-s changeover delay was satisfied). The changeover delay prevented a response from producing food until one second had elapsed since the first response upon a key after changing over from the other key. Side keys were darkened during food delivery and illuminated again five seconds after food had been delivered.

\section{Sessions}

The first session of each condition lasted only 36 food deliveries. The second session began immediately following the end of the first session. After the $36^{\text {th }}$ food delivery in this second session, all lights were extinguished and shortly thereafter the pigeon was returned to its 
home cage and fed if necessary. Approximately 23 hours later, the pigeon was placed back into the chambers and began in blackout. The side keys were then illuminated and the session continued for another 36 food deliveries. Excepting the first and last session of each condition, each session lasted for 72 food deliveries across two calendar days. In other words, there was an overnight break after the $36^{\text {th }}$ food delivery in a session, but no break between the $72^{\text {nd }}$ food delivery of one session and the start of the next session. This pattern continued until the final session of a condition. The final session began immediately after the previous session and only lasted until 36 food deliveries had been obtained. Sessions were terminated if 70 minutes elapsed before a total of 72 food deliveries were produced each day.

In each session, food was delivered to the left and right key according to four possible ratios (shown as left key:right key): 1:5, 1:2, 2:1, and 5:1. Depending on the ratio that was operating, the computer assigned either four-sixths or five-sixths of the food deliveries to the same side key. When the ratio was 1:5, out of every six intervals, the computer selected the left key for one food delivery and the right key for five food deliveries; the same was true for the 5:1 ratio, except that the left key was selected five times and the right key once. Similarly, when the ratio was $1: 2$ or $2: 1$, the computer selected the left or right key, respectively, for 2 deliveries and the opposing key for 4 deliveries.

The ratio in effect for each session was determined according to two randomly generated binary sequences shown in Table 1. These sequences were obtained by downloading two series of random digits generated by a radioactive decay process (retrieved on 17 March, 2015 from http://www.fourmilab.ch/hotbits/). The session column shows which two numbers from the sequence columns were used to determine the ratio of food delivery for each session in a condition. Depending on the condition, one of the sequences was used to determine the relative 
ratio of food delivery (whether food would be delivered two thirds or five sixths of the time to the same key) and the other sequence determined the location of the key (either left or right) that delivered the larger amount of food. In the unsignaled condition, sequence 1 determined the relative ratio of food delivery, whereas sequence 2 determined the key location; the inverse was true for the signaled condition.

For the sequence determining the relative ratio of food delivery, shown in Table 1, sessions with a 0 either had a 1:5 or 5:1 ratio in effect. Sessions with a 1 had a 1:2 or 2:1 ratio in effect. For the sequence determining key location, sessions with a 0 had more food delivered to the right key. Sessions with a 1 had more food delivered to the left key. For example, to determine the ratio in effect for a pigeon in the first session of the unsignaled condition, the numbers from the first row of the Sequence 1 and 2 columns were used. The 1 from Sequence 1 shows that the ratio was either 1:2 or 2:1. The 0 from Sequence 2 shows that more food was delivered to the right key. Therefore, a 1:2 ratio was in effect for the first session.

\section{Conditions}

There were two conditions that differed in how the change from one session to the next was signaled. In the unsignaled condition, the keys were illuminated white throughout every session and during the transition between sessions; there was no change in stimuli across sessions. The potential change in the number of food deliveries assigned to each side key in a block was the only discriminative signal that a new session was beginning. During the signaled condition, a stimulus change accompanied the change from one session to the next. The color of the keys alternated between red and green across sessions throughout the condition. The keys were illuminated red in odd-numbered sessions and green in even-numbered sessions. At the 
start of a new session, the key color switched immediately. The rate of food delivery ratio and the color of the keys remained the same throughout each session.

Figure 2 illustrates the programmed food delivery ratios for both conditions. Sessions are displayed along the horizontal axis while ratios are displayed upon the vertical axis. The ratios in effect for each session were derived from the randomly generated binary sequences shown in Table 1.

Table 2 outlines the order of conditions, and the number of sessions spent in each condition, for each pigeon. Except for Pigeon 105, all pigeons experienced 124 sessions in total because each condition lasted 31 sessions. Pigeon 105 experienced the unsignaled condition followed by the signaled condition. However, a procedural error caused all food to be delivered to the right key during the last five days in the first unsignaled replication, therefore only 26 sessions of useable data were collected from that condition. Pigeon 105 was then moved to the signaled condition for four days. To ensure response allocation was stable in the unsignaled replication, Pigeon 105 was changed back to the unsignaled condition for seven days. Because response patterns in those seven sessions were comparable to response patterns in the previous seven sessions, Pigeon 105 was moved to the replication of the signaled condition at that point.

\section{Data Analysis}

Several analyses were conducted in order to answer the three experimental questions outlined above. In order to examine stable transitional behavior without interference from previous conditions, all analyses are based on data from the last 20 sessions from each condition. This decision was based on precedent established in previously published, similar research (Kyonka \& Grace, 2007; 2008; 2009). 
To answer Question 1, how does response allocation adapt when sessions are run backto-back with no break between them?, I fit Equation 1 to log response ratios from the second half of sessions in each condition. Response ratios are the number of pecks to the left key from the second half of a single session, divided by the number of pecks to the right key from the same period. Response ratios were log transformed. With log-transformed response ratios, 0 represents equal responding between the two keys; positive ratios represent higher rates of responding to the left key and negative ratios represent a higher response rate to the right key. In prior research using similar procedures (Kyonka \& Grace, 2007; 2009; 2010), pigeon’s response allocations typically stopped changing about halfway through the session. Responding during the second half is thus a more stable measure of response allocation. If log response ratios were sensitive to food delivery ratios, the slope of Equation 1 would be greater than zero. T-tests were conducted to determine whether the slopes were statistically significantly greater than zero for each pigeon.

To answer Question 2, to what extent do contingencies retain control over choice following an overnight break in the middle of the session?, a measure of response allocation must be calculated for several time points over the course of the entire session. Sessions were separated into 12 blocks containing 6 intervals each. Response ratios in each block were further separated according to the food delivery ratio in effect. If response allocation was not sensitive to food delivery ratios at the start of the session, then log response ratios would be near 0 and undifferentiated according to food delivery ratios. If sensitivity increased by the end of the session, then response ratios would be differentiated according to the food delivery ratios as the sessions progressed. If control by food delivery ratios was maintained following the overnight, mid-session break, response ratios would remain differentiated following the break. If the break was too long a retention interval for pigeons to remember food delivery ratios, estimates of 
sensitivity would reset to 0 following the break in block 7. Repeated measures analyses of variance (ANOVA) were conducted to determine whether response ratios changed systematically over the course of a session.

Finally, I used a lag analysis (see Equation 2) to answer Question 3, how does cueing the change from one session to the next impact the way in which choice adapts?. I obtained estimates of sensitivity to Lag 0 and Lag 1 ratios in the signaled and unsignaled conditions by conducting separate multiple regressions of log response ratios from each block in each condition on Lag 0 and Lag 1 food delivery ratios. If response ratios became sensitive to food delivery ratios with increased exposure, then estimates of Lag 0 sensitivity should increase across the session. If control by food delivery ratios carried over from one session to the next, then Lag 1 sensitivity would be greater than zero at the start of the session. However, if control by the previous food delivery ratio did not carry over across sessions, then estimates of Lag 1 sensitivity would be near zero throughout the entire session. Repeated measures ANOVAs were conducted to determine whether Lag 0 and Lag 1 sensitivity changed systematically over the course of a session.

\section{Results}

Response allocation was sensitive to food delivery ratios in both conditions; it adapted when sessions were run back-to-back with no break between them (Question 1). Figure 3 shows log response ratios as a function of log food delivery ratios for individual pigeons during the signaled (left panels) and unsignaled (right panels) conditions. Each data point represents performance from a single session. Data were pooled across the initial presentation of a condition and the replication. Dashed lines and accompanying equations represent fits of Equation 1. Estimates of sensitivity are bolded in each equation. To identify slopes that 
characterized systematic relations between log response ratio and log food delivery ratio, $t$-tests were conducted on slopes against a null hypothesis of $a=0$. All $t$-scores had 39 degrees of freedom and were between 4.76 and 16.56 with all ps less than .001. In each case, sensitivity was significantly greater than zero. When food delivery ratios changed unpredictably every 72 intervals, response allocation was sensitive to food delivery ratios, even with mid-session, overnight breaks after the $36^{\text {th }}$ interval. Log response ratios were a positive function of log food delivery ratios, consistent with responding observed on most concurrent VI VI schedules.

Figure 3 shows response allocation from the second half of each session, but does not illustrate how response allocation changed over time. Measuring changes in response allocation over time is necessary to quantify how much control contingencies retain over choice following an overnight break in the middle of the session (Question 2). To illustrate the changes in response allocation as a session progressed, Figure 4 shows log response ratios as a function of blocks in the session for each pigeon in the signaled and unsignaled conditions. To determine how response allocation changed across the session, each session was separated into 12 blocks containing 6 intervals each. Log response ratios in each condition were grouped by the log food delivery ratio in effect for each session. Y coordinates of each data point are the mean of all log response ratios from the same block with the same ratio of food delivery in effect. Because there were no statistically significant differences between the initial condition and replication in either condition (described below), response ratios were averaged across replications. The bottom panels of Figure 4 shows group data averaged across all four subjects. In block 1 of both conditions, response allocation was not significantly different in the four food delivery ratios. Some pigeons allocated more responses to the key with a higher rate of reinforcement early in the session, but there was little differentiation between the specific ratios in effect. Responding 
under the four food delivery ratios gradually differentiated as the session progressed, and was distinguishable by visual inspection in block 6 and all later blocks for most pigeons. As predicted by Equation 1, more responding occurred on the left key relative to the right key when the 2:1 and 5:1 ratios were in effect, whereas relatively more responding occurred to the right key when the 1:2 and 1:5 ratios were in effect. Relatively more responses were allocated toward the richer key during 1:5 and 5:1 ratios compared to $1: 2$ and 2:1 ratios.

For a quantitative comparison of response allocation for each food delivery ratio from each block, log response ratios were entered into a 4-way repeated measures ANOVA with replication, condition, food delivery ratio, and block as factors. Table 3 contains the $F$-scores, $p$ values, $\eta_{p}{ }^{2}$, and Mauchley’s $\chi^{2}$ (when sphericity was violated) for each main effect and interaction. Because this information can be found in the table, these scores will not be reported for non-significant effects below. The main effect of food delivery ratio was statistically significant, $F(1.15,3.44)=41.66, p<.001, \eta_{p}{ }^{2}=.93$, confirming that response allocation differed depending upon the food delivery ratio in effect. ${ }^{1}$ This result aligns with the finding, shown in Figure 3, that response allocation was sensitive to food delivery ratios. The block $\mathrm{x}$ food delivery ratio interaction was significant, $F(2.14,6.42)=10.40, p<.001, \eta_{p}{ }^{2}=.78$.

Differences in log response ratios for different food delivery ratios increased as exposure to those food delivery ratios increased. The main effect of block was not statistically significant, which shows that increases and decreases in response ratios when food delivery ratios were positive or

\footnotetext{
${ }^{1}$ Mauchly's test of sphericity indicated that the assumption of sphericity was violated in several interactions involving the effect of ratio; all $\chi^{2}$ with degrees of freedom of 5 or 560 ranged from 12.22 to 1056.08 , all ps <.05. For each F-ratio that violated sphericity, Greenhouse-Geisser transformations were used to correct for this violation.
} 
negative, respectively, canceled each other out. Finally, the main effect of replication was not statistically significant. No interactions involving replication were statistically significant. There were no statistically significant differences between the initial presentation and the replication of the conditions.

Control of response ratios by current food delivery ratios was not affected by signaling the change from one session to the next. In the ANOVA described in the previous paragraph, the main effect of condition was not statistically significant. Additionally, no interactions involving condition were significant. When log response ratios were compared in this way, it appears that signaling the change in food delivery ratios did not affect response allocation at all. However, this analysis did not take food delivery ratios from previous sessions into account. Response allocation may be sensitive to previous food delivery ratios without significantly altering log response ratios.

In addition to estimating sensitivity to food delivery ratios that were currently in effect for each session, a lag analysis (see Equation 2) was used to estimate the extent to which control by previous ratios carried over from one session to the next. Estimates of Lag 0 and Lag 1 sensitivity were obtained using multiple linear regression. Log response ratios were generated for responding in each block of the last 20 sessions of both replications of the signaled and unsignaled conditions for each pigeon and entered into the multiple regression as dependent variables. For each log response ratio, the log food delivery ratio from the same session (Lag 0) and the log food delivery ratio from the previous session (Lag 1) were entered as predictor variables. A total of 960 log response ratios were calculated for each pigeon, one for each of the 12 blocks of the last 20 sessions for each of four conditions. Forty-eight regressions were conducted for each pigeon subject. Each regression consisted of the 20 log response ratios from a 
particular block in each condition. The unstandardized beta weights generated for the two predictor variables (Lag 0 and Lag 1 food delivery ratios) are $a_{0}$ and $a_{1}$ in Equation 2.

Evaluating changes in these parameter estimates over the course of the session may reveal functional relations that were not clear from a direct analysis of log response ratios.

Comparing Lag 0 and Lag 1 estimates of sensitivity for separate regressions on log response ratios from signaled and unsignaled conditions allowed me to determine how cueing the change from one session to the next impacted choice adaptation (Question 3). Lag 0 sensitivity increased across the session during both the signaled and unsignaled conditions. Figure 5 shows sensitivity parameter estimates for Lag 0 and Lag 1 obtained from regressing log response ratios on $\log$ food delivery ratios for the signaled and unsignaled conditions for each subject. The bottom panel shows group data, wherein response ratios were pooled across all subjects. The Yaxis shows estimates of sensitivity (unstandardized beta weights generated by the regression), and blocks of the session are displayed along the $\mathrm{X}$-axis. Lag 0 sensitivity from each pigeon was entered into a repeated measures ANOVA with block, condition, and replication as withinsubjects factors. Table 4 shows $F$-scores, $p$-values, and $\eta_{p}{ }^{2}$ for each main effect and interaction for this ANOVA. Because this information can be found in the table, these scores will not be reported for non-significant effects below. The main effect of block was significant, $F(11,33)=$ 35.60, $p<.001, \eta_{p}{ }^{2}=.92$. Consistent with the increased differentiation in log response ratio by programmed log food delivery ratio as a function of block, Lag 0 sensitivity increased as the session progressed. The block x condition interaction was also significant, $F(11,33)=2.35, p$ $=.03, \eta_{p}{ }^{2}=.44$. Estimates of Lag 0 sensitivity changed across the session differently in the two conditions. The main effect of condition was not significant, showing that there was no difference in Lag 0 sensitivity between the signaled and unsignaled conditions. The main effect 
of replication and the interactions involving replication were not statistically significant. There were no differences in responding during the initial condition and the replication for either the signaled or unsignaled condition.

Lag 0 sensitivity increased by the end of the first half of the session, just before the overnight break, and returned to that same level, before increasing further, early in the second half. Lag 0 sensitivity estimates were lowest at the start of the session, confirming that response allocation was least sensitive to the current food delivery ratios at the beginning of each session. Lag 0 sensitivity was higher during block 6 than most of the previous blocks, and was similar to sensitivity early in the second half of the session (besides block 7). Additionally, estimates of Lag 0 sensitivity were highest at the end of the session, showing that response allocation did become sensitive to the current food delivery ratios. Finally, Lag 0 sensitivity was lower during block 7 than in block 6 for almost all pigeons in both conditions, but sensitivity was higher following the overnight break compared to the start of the session. For pigeon 105, Lag 0 sensitivity was higher in block 7 than in block 6 in both conditions. Differences in estimates of Lag 0 sensitivity are consistent with the changes in response allocation shown in Figure 4.

While estimates of Lag 0 sensitivity are consistent with earlier results, estimates of Lag 1 sensitivity show the extent to which control by log food delivery ratios carried over from one session to the next. Lag 1 sensitivity was relatively high during blocks 1 and 7, but lower in every other block. For a quantitative characterization of the differences in Lag 1 sensitivity at different blocks in a session, estimates of Lag 1 sensitivity obtained from multiple regressions for individual pigeons were entered into a repeated measures ANOVA with block, condition, and replication as within-subjects factors. Table 5 shows $F$-scores, $p$-values, and $\eta_{p}{ }^{2}$ for each main effect and interaction for this ANOVA. Because this information can be found in the table, these 
scores will not be reported for non-significant effects below. The main effect of block was

statistically significant, $F(11,33)=14.48, p<.001, \eta_{p}{ }^{2}=.83$. The block $x$ condition interaction was also statistically significant, $\mathrm{F}(11,33)=6.56, p<.001, \eta_{p}{ }^{2}=.69$. Lag 1 sensitivity changed over the course of the session, and this change was different between the signaled and unsignaled conditions. The main effect of condition was not statistically significant. The main effect of replication and the interactions involving replication were not statistically significant.

Lag 1 sensitivity was high at the start of the session and following the overnight break, especially in the unsignaled condition, but was low throughout the rest of the session. Figure 6 shows comparisons of Lag 1 sensitivity in the signaled and unsignaled conditions from block 1 (top panels) and block 7 (bottom panels). In all cases, estimates of Lag 1 sensitivity were higher in the unsignaled condition than in the signaled condition. This suggests that control by previous ratios did carryover from one session to the next, especially during the unsignaled condition. Following the overnight break, Lag 1 sensitivity briefly increased during the unsignaled condition but remained low during the signaled condition. In both conditions, Lag 1 sensitivity decreased as the session progressed.

\section{Discussion}

In this concurrent VI VI schedule procedure, food delivery ratios were 5:1, 2:1, 1:2, or 1:5, determined across sessions by independent random series. Changes in the food delivery ratio occurred in the middle of the day and carried over to the next day following an overnight break. The session change was not cued during the unsignaled condition, but was cued by a change in the color of the key lights during the signaled condition. The first objective of this experiment was to determine the extent to which response allocation became sensitive to food delivery ratios when sessions were run back-to-back with no break between them (Question 1). Consistent with 
predictions by the generalized matching law, log food delivery ratios from the current session controlled response allocation in both conditions. This was demonstrated by fitting the generalized matching law to response allocation from the latter half of sessions (see Figure 3). The second objective was to examine whether contingencies would retain control over response allocation following a mid-session, overnight break (Question 2). Log response ratios and estimates of sensitivity (see Figures 4 and 5) showed that pigeons continued to respond to the current contingencies following the overnight break. The final objective of this study was to investigate how signaling the change in sessions altered the way response allocation adapted to the new contingencies (Question 3). Lag analyses showed that response allocation adapted to current session food delivery ratios whether or not the change in sessions was signaled (see Figure 5). During the unsignaled condition, where the change in ratios was not cued, control by the previous ratio carried over into the new session. Sensitivity to the immediately previous ratio was near zero during the signaled condition, demonstrating that pigeons would adaptively forget past ratios when a change is cued.

In this experiment, a stimulus change in the form of changing key colors facilitated adaptive forgetting of previous ratios in pigeons. This is relevant to operant conditioning researchers who study behavior in dynamic environments. Previous research (e.g., Schofield \& Davison, 1997; Grace, Bragason, \& McLean, 2003) has found that non-human animals would forget past food delivery ratios when an overnight break accompanied the change in ratios. Schofield and Davison (1997) manipulated log reinforcement ratios every session across calendar days and found that pigeons would forget previous ratios at the start of each session response allocation was not sensitive to previous ratios. The change in ratios always occurred at the same time as the overnight break between sessions, so it could have been that the break was 
too long for contingencies to retain control over response allocation. Alternatively, it is possible the overnight break was a cue to forget past ratios. In the present experiment, response allocation remained sensitive to log food delivery ratios following an overnight break. This suggests that the overnight break in Schofield and Davison's study functioned as a cue to adaptively forget past ratios.

Log response ratios from the present experiment showed that pigeons remembered contingencies following a break in both conditions, and a lag analysis revealed evidence of adaptive forgetting when the session change was cued. Adaptive forgetting has occurred following a stimulus change, in the present experiment, and following an overnight break, as in Schofield and Davison’s (1997) study and other rapid acquisition experiments (Grace, Bragason, \& McLean, 2003; Grace \& McLean, 2006; Kyonka \& Grace, 2007). It appears that an overnight break is a sufficient cue for animals to forget past ratios, but it is not a necessary cue. However, some kind of signal is necessary for adaptive forgetting to occur. Pigeons did not adaptively forget past ratios when the session change was not cued by a stimulus change.

The finding that control by schedules of reinforcement can be retained overnight is consistent with an experiment performed by Christensen and Grace (2009). Christensen and Grace manipulated the terminal link delays of a concurrent chain schedule predictably in an ascending and descending series across sessions. During the ascending series, one of the terminal link delays became progressively longer from one session to the next. During the descending series, the terminal link delay was progressively shortened. Response allocation tracked the predictable changes in terminal link delay at the start of sessions, supporting the claim that pigeons can remember past ratios following an overnight break even when the ratios are changing. 
In the present experiment, food delivery ratios changed at approximately the same time every day, but the new food delivery ratio was unpredictable for the pigeon. When the change was signaled, it did not cue a specific ratio but instead cued a possible change in ratios. In a sense, the cue signaled the pigeons to forget old contingencies and prepare to learn the upcoming contingency. This could have implications for how animal behavior adapts to dynamic environments outside of the lab. For example, a person may be driving down the highway and see a sign that says "Expect changes in traffic." This sort of signal does not specify the upcoming contingency, as the speed of traffic may increase or decrease, but it does prepare the driver to adapt to whatever new traffic contingencies may occur. This finding may also be relevant to the instructional design of schools. In most school settings, various subjects are taught at different times of day, by different teachers, or in different classrooms. Findings from the present study and previous research has shown that differing dimensions of stimulus changes (e.g., a break from the session, changes in key color) can facilitate adaptive forgetting in similar ways. This supports the practice of having some clear cue, such as a break between classes, in order to help behavior adapt to upcoming contingencies.

Further research could extend the findings of the present study in several directions. It was found that response allocation adapted to sudden changes in contingencies and remained sensitive to contingencies following a long break, but this may not occur with dimensions of behavior other than response allocation. Kyonka and Grace (2007) found that choice and timing behavior may be linked by a common process. If this is the case, then temporal aspects of behavior may also adapt and remain sensitive to contingencies in a similar procedure. Further research is warranted to identify whether other dimensions of behavior, such as timing, can adapt to constantly changing contingencies with a long break in-between sessions. 
The signals in the present experiment, as described above, signaled a change in the contingencies but did not specify what those changes were. Future work could examine the extent to which adaptive forgetting and remembering are observed when contingencies are signaled with specific cues in a similar procedure. Pigeons immediately forgot past ratios following the signal in the present study, but there was no opportunity to observe whether they would adaptively remember past contingencies that had been paired with a specific stimulus. If pigeons remembered contingencies in this way, then response allocation would be immediately sensitive to those contingencies following the signal.

The overnight break is a sufficient cue for adaptive forgetting, and most non-human animal research is conducted with one session per day at about the same time every day with a long overnight break between sessions. In these situations, the circadian structure of the sessions may come to control some aspects of behavior. Potential effects that consistent session scheduling has over behavior warrants further attention. Studies could be conducted to analyze behavior in sessions that are conducted multiple times per day or at different times across days. This could be compared to behavior during traditional sessions that are scheduled at the same time every day. While these studies would not challenge the results from studies using the traditional approach, there is more we could learn about behavior by manipulating the circadian structure of an experiment. 


\section{References}

Baum, W. M. (1974). On two types of deviation from the matching law: bias and undermatching. Journal of the Experimental Analysis of Behavior, 22, 231-242.

Baum, W. M. (1979). Matching, undermatching, and overmatching in studies of choice. Journal of the Experimental Analysis of Behavior, 32, 269-281.

Baum, W. M., \& Rachlin, H. C. (1969). Choice as time allocation. Journal of the Experimental Analysis of Behavior, 12, 861-874.

Brown, G. E., Ferrari, M. C. O., Malka, P. H., Oligny, M., Romano, M., \& Chivers, D. P. (2011). Growth rate and retention of learned predator cues by juvenile rainbow trout: fastergrowing fish forget sooner. Behavioral Ecology and Sociobiology, 65, 1267-1276.

Christenson, D. R., \& Grace, R. C. (2009) Response allocation in concurrent chains when terminal-link delays follow an ascending and descending series. Journal of the Experimental Analysis of Behavior, 91, 1-20.

Davison, M., \& McCarthy, D. (1988). The matching law: A research review. University of Hillsdale, NJ: L. Erlbaum.

Epp, K. J. (2013) Threat sensitivity in the San Marcos salamander: effects of predator diet and prey experience. Behaviour, 150, 617-634.

Ferrari, M. C. O., \& Chivers, D. P. (2013). Temporal dynamics of information use in learning and retention of predator-related information in tadpoles. Animal Cognition, 16, 667-676.

Fleshler, M. \& Hoffman, H. S. (1962). A progression for generating variable-interval schedules. Journal of the Experimental Analysis of Behavior, 5, 529-530.

Grace, R. C., Bragason, O., \& McLean, A. P. (2003). Rapid acquisition of preference in concurrent chains. Journal of the Experimental Analysis of Behavior, 80, 235-252. 
Grace, R. C., \& Hucks, A. D. (2013). The allocation of operant behavior. In Madden, G. J., Dube, W. V., Hackenberg, T. D., Hanley, G. P., \& Lattal, K. A. (Eds.), APA handbook of behavior analysis, Vol. 1: Methods and principles (307 -337). Washington, DC, US: American Psychological Association.

Grace, R. C., \& McLean, A. P. (2006). Rapid acquisition in concurrent chains: evidence for a decision model. Journal of the Experimental Analysis of Behavior, 85, 181-202.

Grace, R. C., \& Nevin, J. A. (1999). Timing and choice in concurrent chains. Behavioural Processes, 45, 115-127.

Herrnstein, R. J. (1961). Relative and absolute strength of response as a function of frequency of reinforcement. Journal of the Experimental Analysis of Behavior, 4, 267-272.

Johnston, J. M., \& Pennypacker, H. S. (2009). Strategies and tactics of behavioral research (3 ${ }^{\text {rd }}$ ed.). New York, NY: Routledge.

Kraemer, P. J., \& Golding, J. M. (1997). Adaptive forgetting in animals. Psychonomic Bulletin \& Review, 4, 480-491.

Kyonka, E. G. E., \& Grace, R. C. (2007). Rapid acquisition of choice and timing in pigeons. Journal of Experimental Psychology: Animal Behavior Processes, 33, 392-408. doi: 10.1037/0097-7403.33.4.392

Kyonka, E. G. E., \& Gace, R. C. (2008). Rapid acquisition of preference in concurrent chains when alternatives differ on multiple dimensions of reinforcement. Journal of the Experimental Analysis of Behavior, 89, 49-69.

Kyonka, E. G. E., \& Grace, R. C. (2009). Effects of unpredictable changes in initial-link duration on choice and timing. Behavioural Processes. doi:10.1016/j.beproc.2008.12.024

Kyonka, E. G. E., \& Grace, R. C. (2010). Rapid acquisition of choice and timing and the 
provenance of the terminal-link effect. Journal of the Experimental Analysis of Behavior, 94, 209-225.

Myers, D. L, \& Myers L. E. (1977) Undermatching: a reappraisal of performance on concurrent variable-interval schedules of reinforcement. Journal of the Experimental Analysis of Behavior, 25, 203-214.

Poling, A., Edwards, T. L., Weeden, M., \& Foster, T. M. (2011). The matching law. The Psychological Record, 61, 313-322.

Schofield, G., \& Davison, M. (1997). Nonstable concurrent choice in pigeons. Journal of the Experimental Analysis of Behavior, 68, 219-232.

Sidman, M. (1960). Tactics of scientific research. New York: Basic Books

Staddon, J. E. R. (1968). Spaced responding and choice: a preliminary analysis. Journal of the Experimental Analysis of Behavior, 11, 669, 682.

Stubbs, D. A., \& Pliskoff, S. S. (1969). Concurrent responding with fixed relative rates of reinforcement. Journal of the Experimental Analysis of Behavior, 12, 887-895.

Subramaniam, S., \& Kyonka, E. G. E. (2016). Environmental dynamics modulate covariation of choice and timing. Behavioural Processes, 124, 130-140.

Wearden, J. H., \& Burgess, I. S. (1982). Matching since Baum (1979). Journal of the Experimental Analysis of Behavior, 38, 339-348. 
Table 1

Random Binary Sequences Used to Determine the Rate of Food Delivery Ratio for both signaled and unsignaled Conditions

\begin{tabular}{|c|c|c|}
\hline Session & Sequence 1 & Sequence 2 \\
\hline 1 & 1 & 0 \\
\hline 2 & 0 & 1 \\
\hline 3 & 0 & 0 \\
\hline 4 & 0 & 1 \\
\hline 5 & 0 & 0 \\
\hline 6 & 0 & 1 \\
\hline 7 & 1 & 0 \\
\hline 8 & 1 & 1 \\
\hline 9 & 1 & 1 \\
\hline 10 & 0 & 0 \\
\hline 11 & 0 & 1 \\
\hline 12 & 1 & 1 \\
\hline 13 & 1 & 0 \\
\hline 14 & 0 & 0 \\
\hline 15 & 1 & 1 \\
\hline 16 & 1 & 1 \\
\hline 17 & 0 & 1 \\
\hline 18 & 0 & 1 \\
\hline 19 & 1 & 1 \\
\hline 20 & 1 & 1 \\
\hline 21 & 1 & 0 \\
\hline 22 & 0 & 0 \\
\hline 23 & 1 & 0 \\
\hline 24 & 0 & 0 \\
\hline 25 & 1 & 0 \\
\hline 26 & 0 & 1 \\
\hline 27 & 0 & 1 \\
\hline 28 & 0 & 0 \\
\hline 29 & 1 & 0 \\
\hline 30 & 1 & 0 \\
\hline 31 & 0 & 1 \\
\hline
\end{tabular}


Table 2

Summary of Experimental Conditions

\begin{tabular}{llc} 
Pigeon & Condition & Sessions \\
\hline 305 & Signaled & 31 \\
& Unsignaled & 31 \\
& Signaled & 31 \\
& Unsignaled & 31 \\
\hline 106 & Signaled & 31 \\
& Unsignaled & 31 \\
& Signaled & 31 \\
& Unsignaled & 31 \\
\hline 306 & Unsignaled & 31 \\
& Signaled & 31 \\
& Unsignaled & 31 \\
& Signaled & 31 \\
\hline \multirow{2}{*}{105} & Unsignaled $^{3}$ & 31 \\
& Signaled & 31 \\
& Unsignaled & 26 \\
& Signaled & 4 \\
& Unsignaled & 7 \\
& Signaled & 31 \\
\hline
\end{tabular}

Note. Only sessions that were appropriately administered are shown in this table.

${ }^{a} A$ procedural error caused all food to be delivered to the right key during the last five sessions of the second unsignaled condition for Pigeon 105. This led to several condition changes before this bird could be moved to the last condition. 
Table 3

Results of a 4-way Analysis of Variance with Log Response Ratios

\begin{tabular}{|c|c|c|c|c|c|}
\hline Effect or Interaction & $\begin{array}{l}\text { Degrees of } \\
\text { Freedom }\end{array}$ & F-Score & $P$-Value & $\eta_{\mathrm{p}}^{2}$ & Mauchley's $\chi^{2}$ \\
\hline Replication & $(1,3)$ & 0.06 & 0.83 & 0.02 & \\
\hline Condition & $(1,3)$ & 0.02 & 0.89 & 0.01 & \\
\hline Food Delivery Ratio & $(1.15,3.44)$ & 41.66 & 0.00 & 0.93 & 6.91 \\
\hline Block & $(1.43,4.29)$ & 0.86 & 0.59 & 0.22 & 18.84 \\
\hline Replication x Condition & $(1,3)$ & 0.41 & 0.57 & 0.12 & \\
\hline Replication x Ratio & $(1.57,4.70)$ & 2.12 & 0.17 & 0.41 & 4.06 \\
\hline Condition x Ratio & $(1.61,4.82)$ & 0.71 & 0.57 & 0.19 & 12.34 \\
\hline Replication x Block & $(2.18,6.53)$ & 0.80 & 0.64 & 0.21 & 24.25 \\
\hline Condition x Block & $(1.43,4.29)$ & 1.30 & 0.27 & 0.30 & 19.83 \\
\hline Ratio x Block & $(2.14,6.42)$ & 10.40 & 0.00 & 0.78 & 942.24 \\
\hline Replication x Condition x Ratio & $(1.20,3.59)$ & 0.35 & 0.79 & 0.11 & 12.22 \\
\hline Replication x Condition x Block & $(2.10,6.31)$ & 1.46 & 0.19 & 0.33 & 22.14 \\
\hline Replication x Ratio x Block & $(2.57,7.72)$ & 0.72 & 0.86 & 0.19 & 1056.08 \\
\hline Condition x Ratio x Block & $(2.16,6.48)$ & 1.13 & 0.31 & 0.27 & 1001.56 \\
\hline $\begin{array}{c}\text { Replication x Condition x Ratio } \\
\text { x Block }\end{array}$ & $(1.85,5.56)$ & 1.15 & 0.29 & 0.28 & 911.17 \\
\hline
\end{tabular}

Note. $\eta_{\mathrm{p}}{ }^{2}=$ partial eta squared. Greenhouse-Geisser transformations for Degrees of Freedom and $F$ Scores are shown where applicable. 
Table 4

Results of a 3-way Analysis of Variance with Estimates of Lag 0 Sensitivity

\begin{tabular}{ccccc} 
Effect or Interaction & $\begin{array}{c}\text { Degrees of } \\
\text { Freedom }\end{array}$ & F-Score & $P$-Value & $\eta_{\mathrm{p}}{ }^{2}$ \\
\hline Replication & $(1,3)$ & 0.31 & 0.62 & 0.09 \\
Condition & $(1,3)$ & 0.16 & 0.72 & 0.05 \\
Block & $(11,33)$ & 35.60 & 0.00 & 0.92 \\
$\begin{array}{c}\text { Replication x } \\
\text { Condition }\end{array}$ & $(1,3)$ & 0.58 & 0.50 & 0.16 \\
$\begin{array}{c}\text { Replication x Block } \\
\begin{array}{c}\text { Condition x Block } \\
\text { Replication x }\end{array}\end{array}$ & $(11,33)$ & 0.49 & 0.90 & 0.14 \\
Condition x Block & $(11,33)$ & 1.44 & 0.20 & 0.32 \\
\hline
\end{tabular}

Note. $\eta_{\mathrm{p}}{ }^{2}=$ partial eta squared. 
Table 5

Results of a 3-way Analysis of Variance with Estimates of Lag 1 Sensitivity

\begin{tabular}{ccccc} 
Effect or Interaction & $\begin{array}{c}\text { Degrees of } \\
\text { Freedom }\end{array}$ & F-Score & $P$-Value & $\eta_{\mathrm{p}}{ }^{2}$ \\
\hline Replication & $(1,3)$ & 0.00 & 0.97 & 0.00 \\
Condition & $(1,3)$ & 7.53 & 0.07 & 0.72 \\
Block & $(11,33)$ & 14.48 & 0.00 & 0.83 \\
$\begin{array}{c}\text { Replication x } \\
\text { Condition }\end{array}$ & $(1,3)$ & 0.57 & 0.51 & 0.16 \\
$\begin{array}{c}\text { Replication x Block } \\
\begin{array}{c}\text { Condition x Block } \\
\text { Replication x }\end{array}\end{array}$ & $(11,33)$ & 0.74 & 0.69 & 0.20 \\
Condition x Block & $(11,33)$ & 0.96 & 0.50 & 0.24 \\
\hline
\end{tabular}

Note. $\eta_{\mathrm{p}}^{2}=$ partial eta squared. 


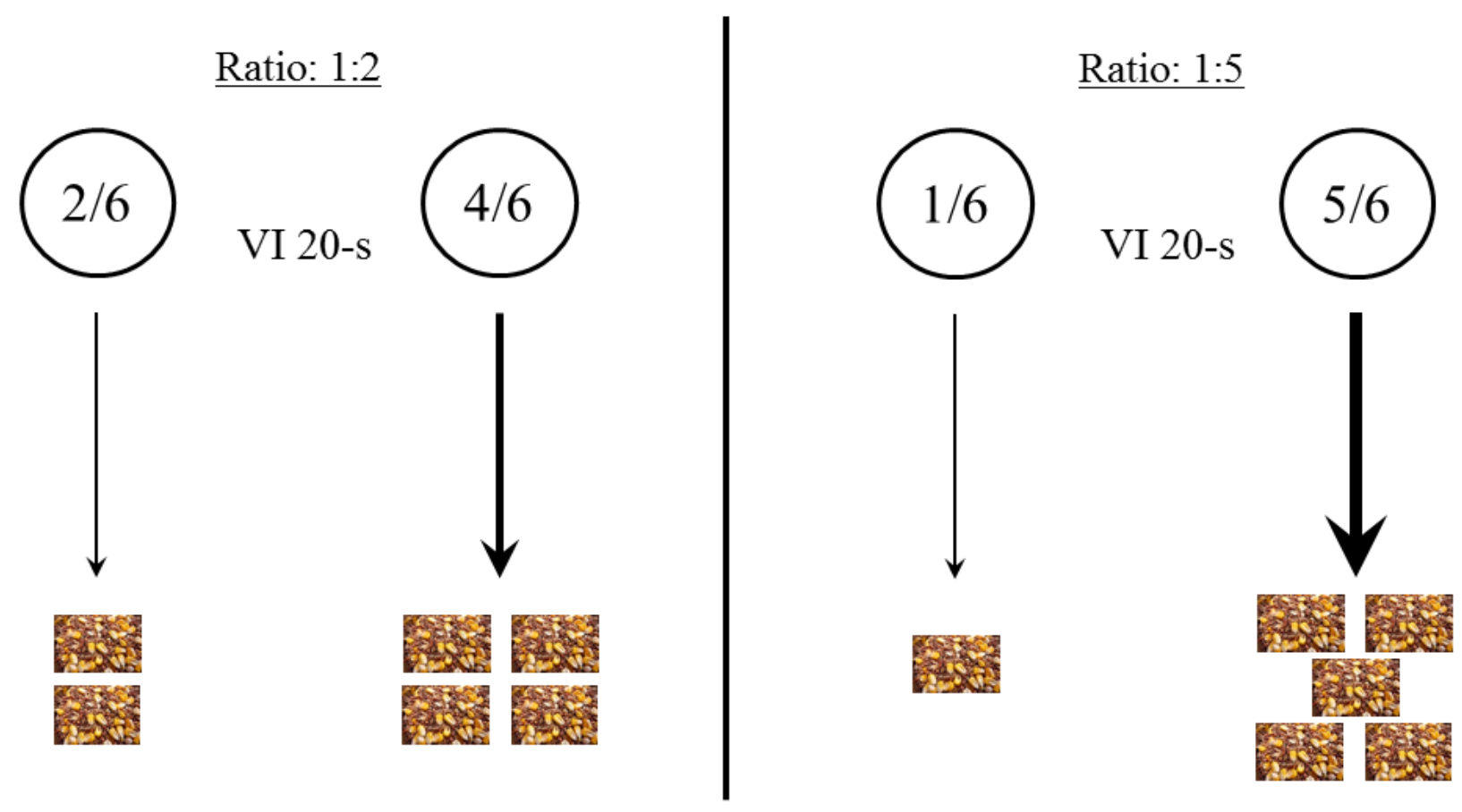

Figure 1. The procedural diagram for an interdependent concurrent VI 20-s VI 20-s schedule during the unsignaled condition. The circles represent side keys that are illuminated white. The fractions inside the keys, and the pictures of food, represent the number of times within a block of 6 intervals that food was delivered to that key. The left side displays a ratio of 1:2 and the right side displays a ratio of 1:5. A single VI 20-s schedule was running during any one interval, and the ratio affected how often that schedule occurred upon each of the keys. Ratios of 2:1 and 5:1 would be similar, except that the number of food deliveries to each key would be swapped. The procedure was identical during the signaled condition, except that the keys would be illuminated either red or green. 


\section{Signaled Condition}

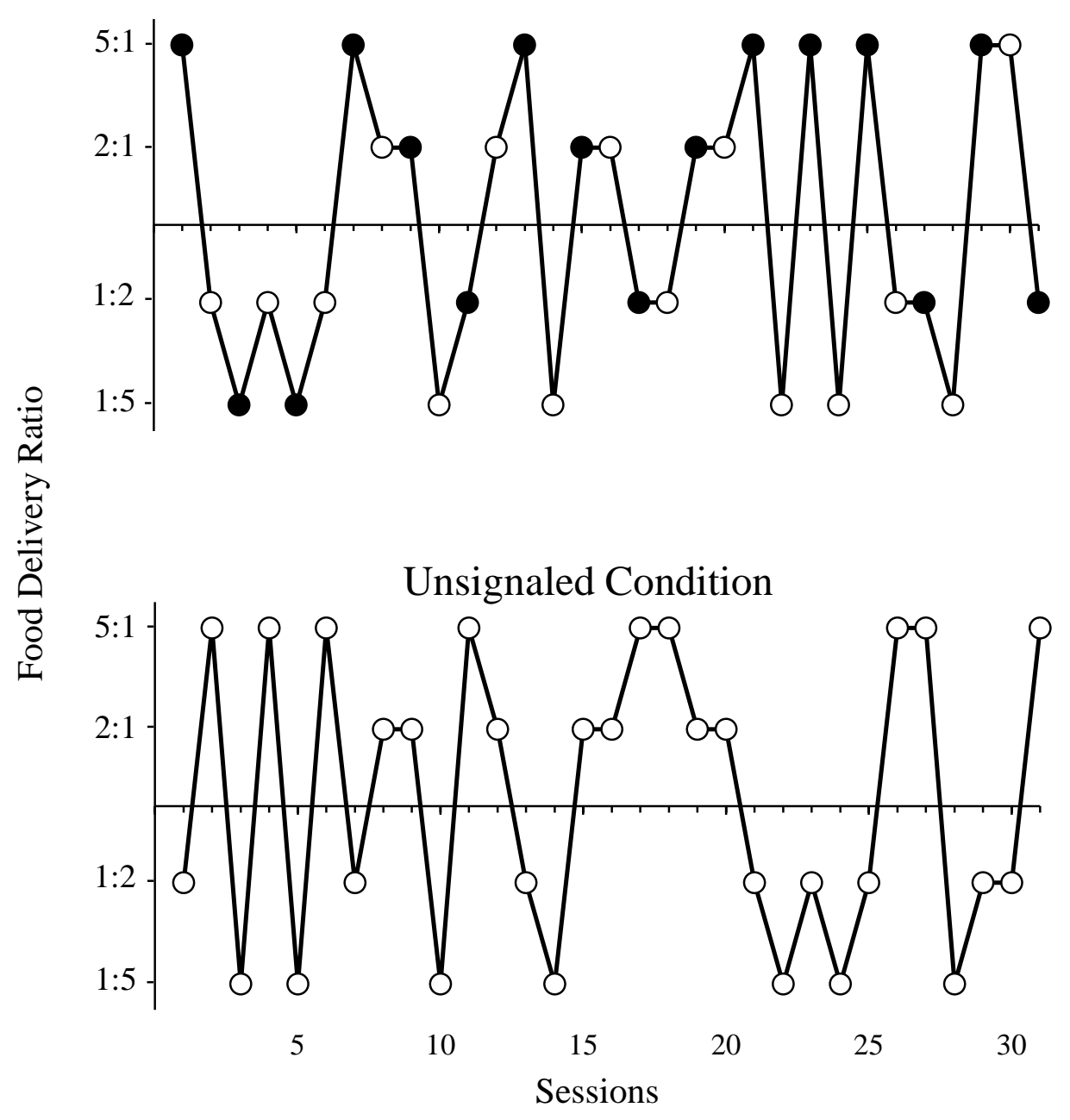

Figure 2. The order in which food delivery ratios were programmed is shown for both the signaled (top) and unsignaled (bottom) conditions. All ratios (shown left key:right key) describe the relative number food deliveries to each key within a session. For the signaled condition, filled and empty circles represent sessions where the keys were illuminated red or green, respectively. 


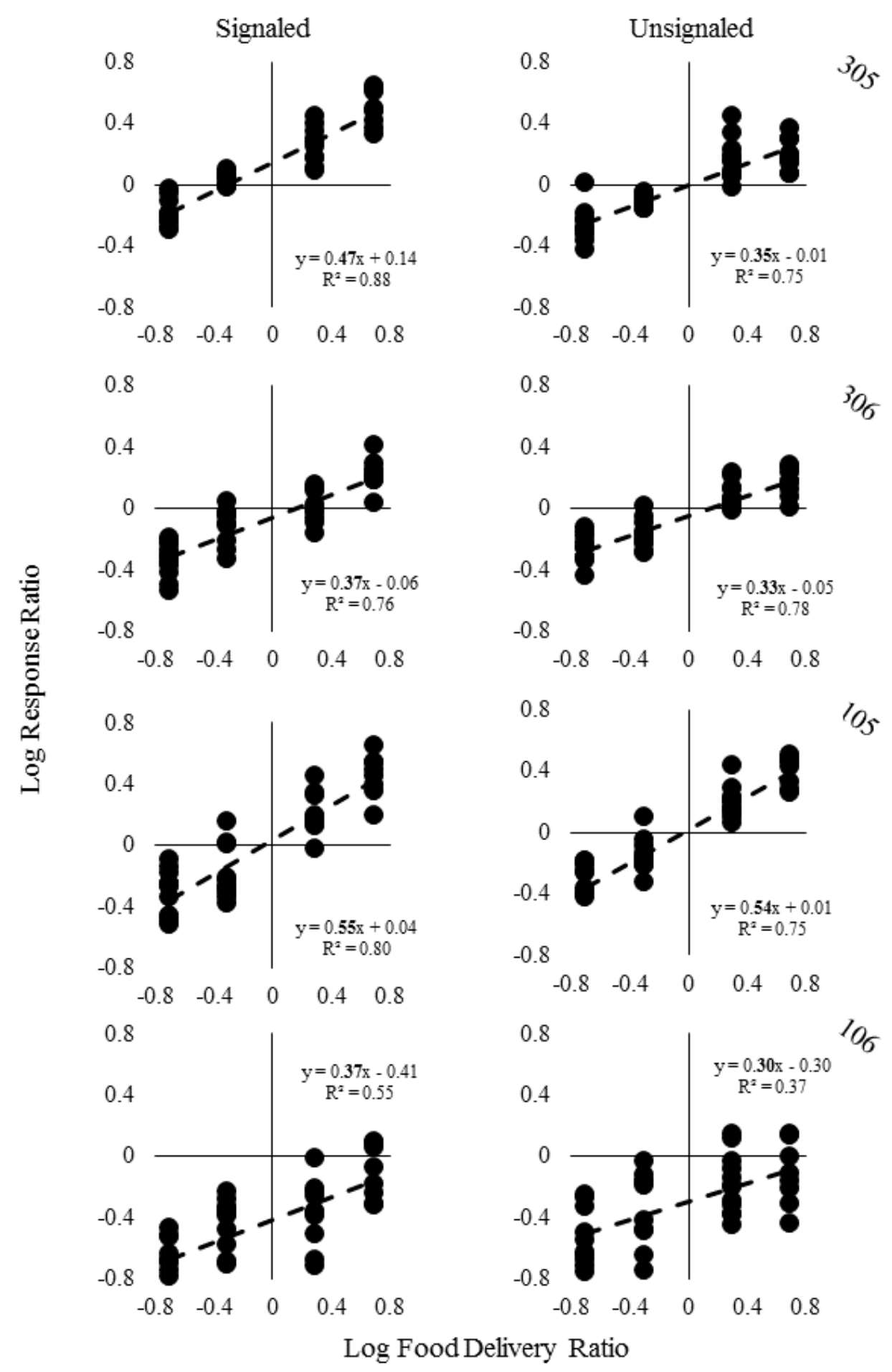

Figure 3. Log response ratios as a function of log food delivery ratios for all subjects. Log response ratios were pooled from the second half of the last 20 sessions of the initial conditions and replications of the signaled and unsignaled conditions. Responding during the signaled 
condition is shown on the left, while responding during the unsignaled condition is shown on the right. Each data point represents performance from a single session. Pigeon IDs are displayed on the right side of the figure. Parameters and variance accounted for $\left(\mathrm{R}^{2}\right)$ by linear regression (dashed lines) are shown. 
Signaled
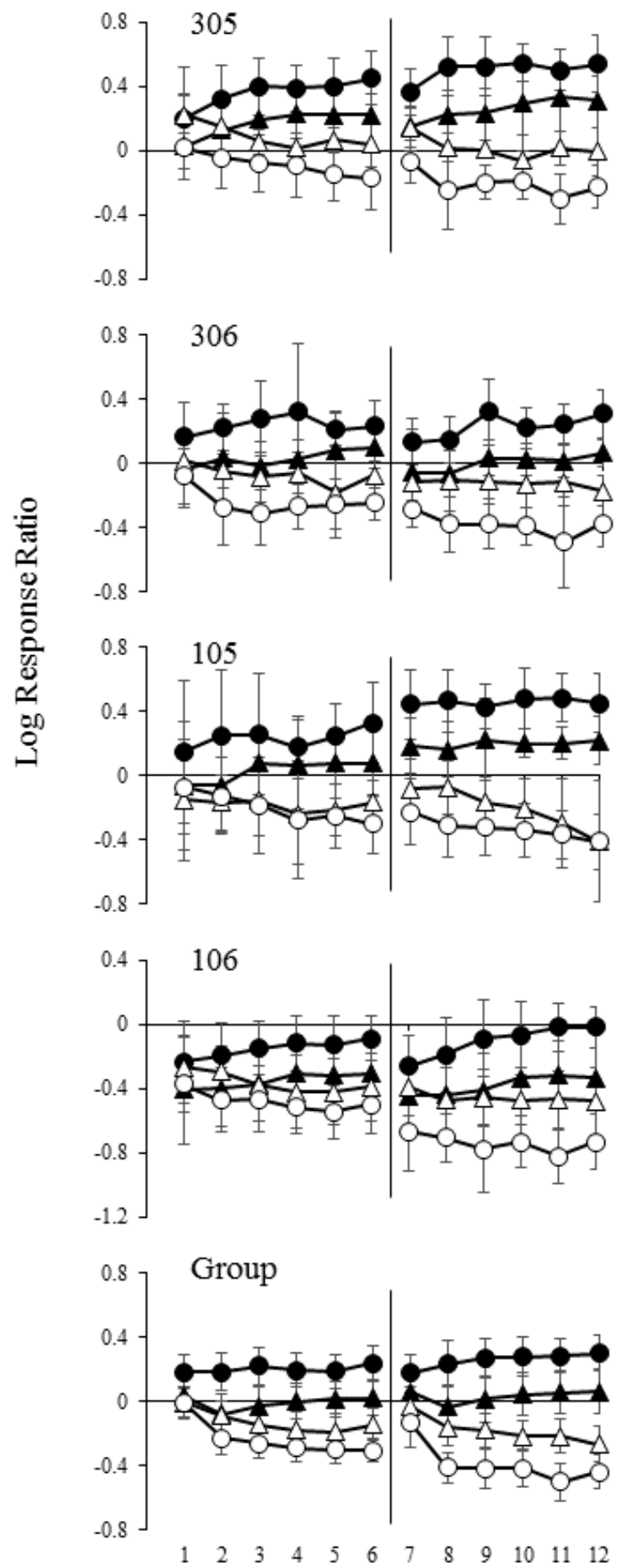

Unsignaled
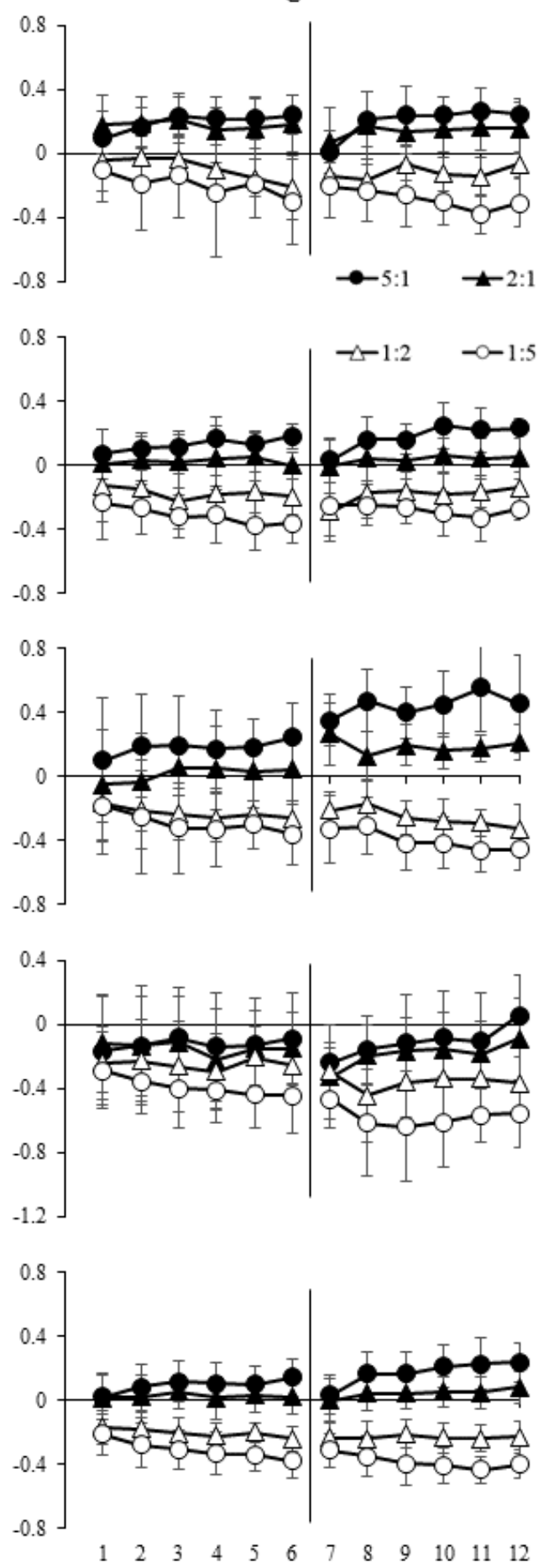

Blocks of 6 Intervals

Figure 4. Log response ratios displayed as a function of blocks of the session during the signaled (left) and unsignaled (right) conditions for each subject. Blocks contain 6 intervals each. 
Each data point represents the mean of all log response ratios from the same block with the same ratio of food delivery in effect. Response ratios were averaged from the initial condition and replication of each condition. The bottom panels show data averaged across all four subjects. Filled circles and triangles show response ratios from sessions when reinforcer ratios were 5:1 and 2:1, respectively. Empty circles and triangles show response ratios from sessions when reinforcer ratios were 1:5 and 1:2, respectively. Error bars represent the standard deviation for individual subjects and standard error for the group data. Vertical lines represent the mid-session overnight break. 
Signaled

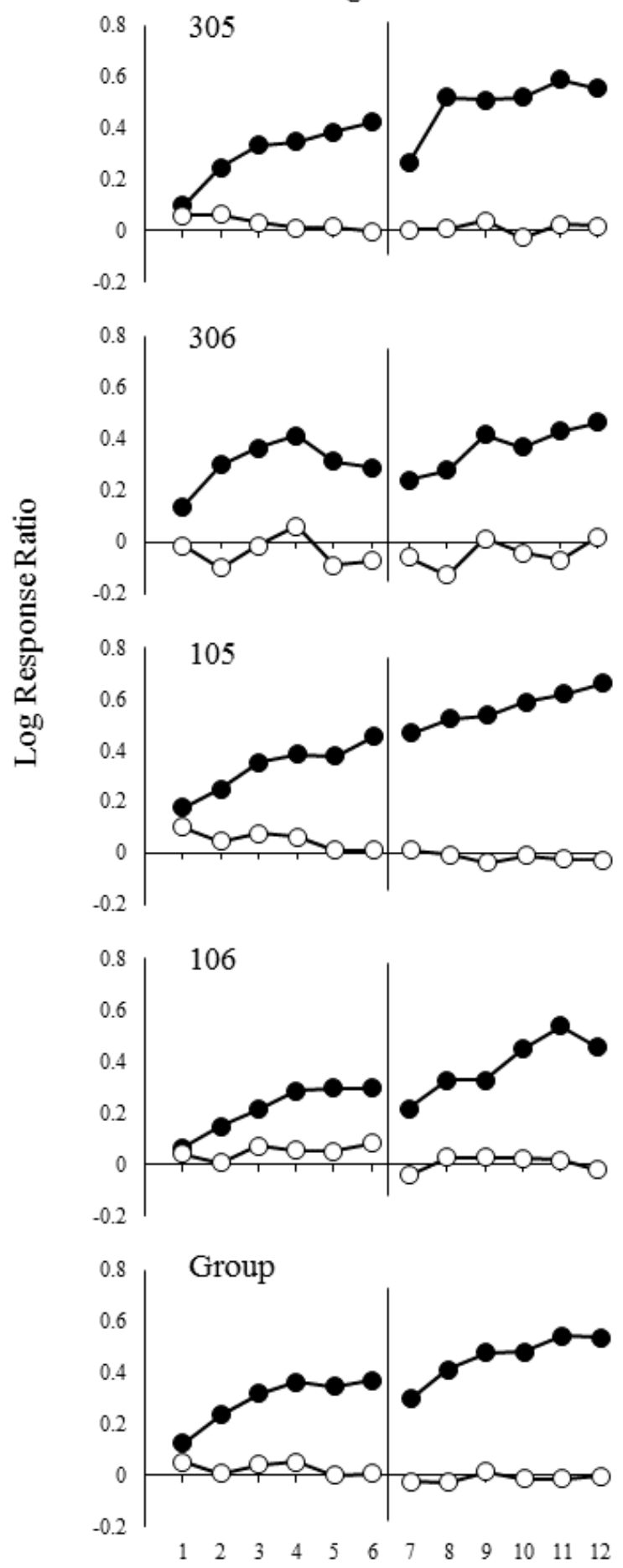

Unsignaled
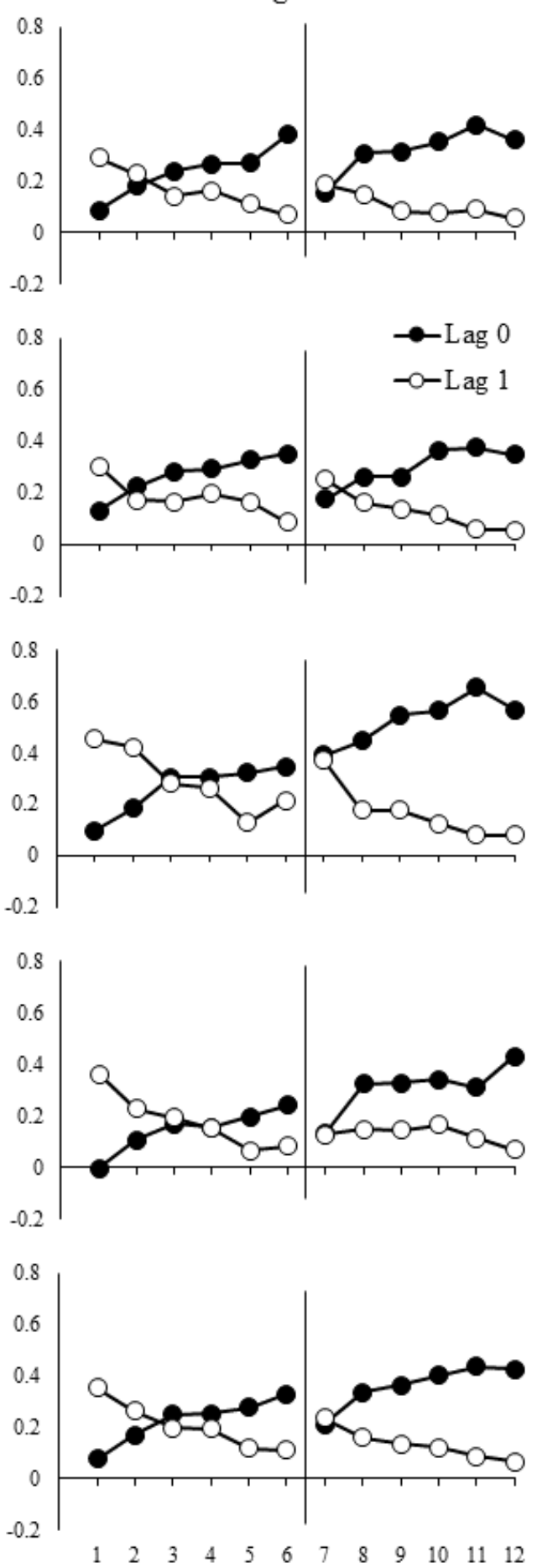

Blocks of 6 Intervals

Figure 5. Sensitivity to log food delivery ratios as a function of blocks of the session in the signaled and unsignaled conditions for each subject. Data were pooled across the initial condition 
and replication of each condition. The bottom panel shows data from all four subjects combined; data were pooled across initial and replication conditions for all subjects. Filled circles represent sensitivity to food delivery ratios lagged 0 sessions relative to responding (Lag 0 sensitivity). Empty circles represent sensitivity to food delivery ratios lagged 1 session relative to responding (Lag 1 sensitivity). Vertical lines represent the mid-session overnight break. 

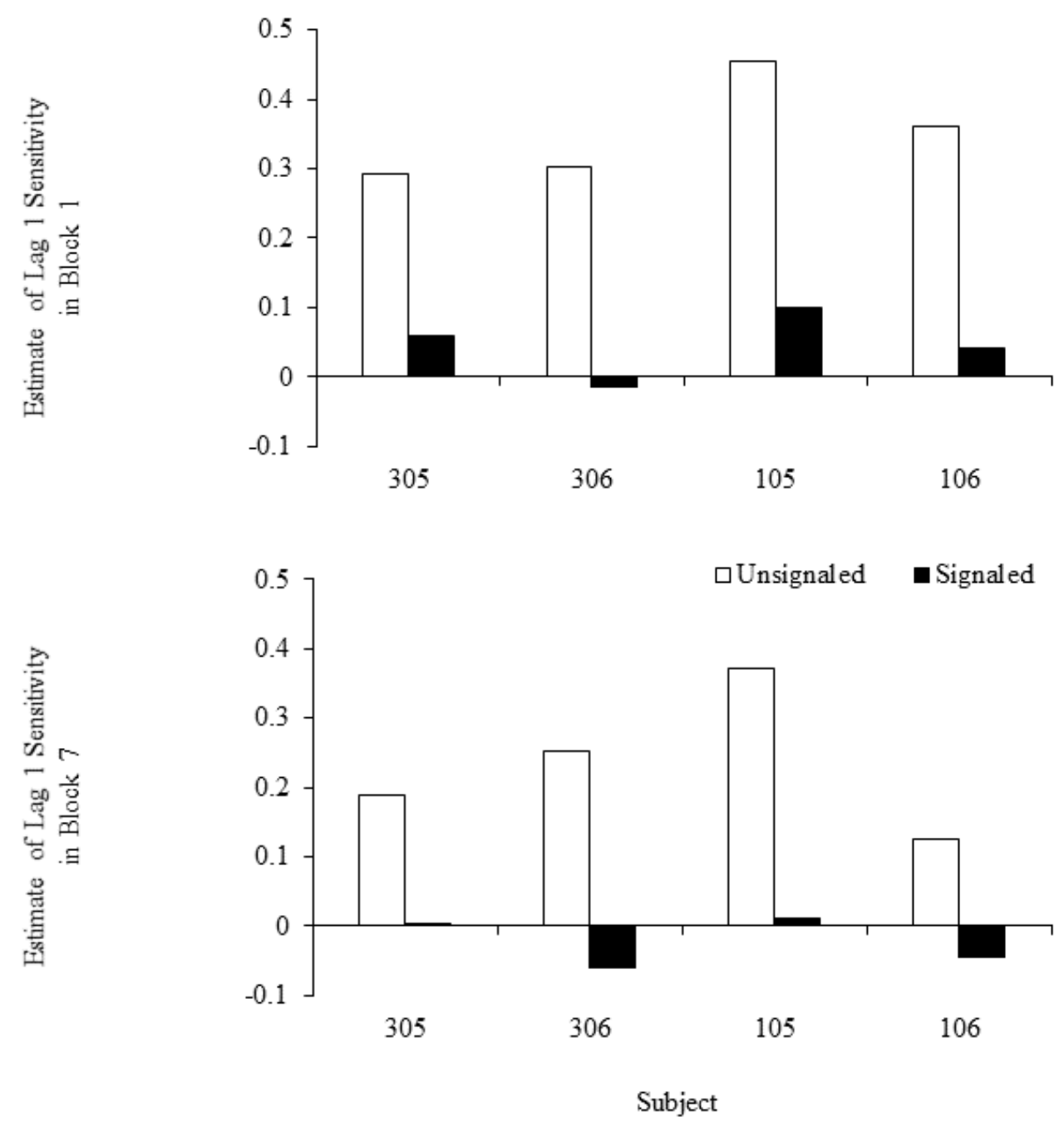

Figure 6. Estimates of Lag 1 sensitivity are compared across the unsignaled and signaled conditions for all subjects. The top panel shows Lag 1 sensitivity estimated from block 1 of the sessions (the beginning of the session), while the bottom panel shows Lag 1 sensitivity estimated from block 7 of the sessions (immediately following the overnight break). Estimates of sensitivity were determined using response ratios pooled across the initial condition and replication of each condition for each subject. White bars show Lag 1 sensitivity during the unsignaled condition, and black bars show Lag 1 sensitivity during the signaled condition. 